\title{
Corrigendum
}

\section{An unsettled debate: Key empirical and theoretical questions are still open - CORRIGENDUM}

\author{
Stefano Vincini, ${ }^{a}$ Yuna Jhang, ${ }^{\text {b }}$ Eugene H. Buder, ${ }^{c, d}$ and Shaun Gallagher ${ }^{d, e, f}$ \\ a UNAM Postdoctoral Fellowships Program, Instituto de Investigaciones Filosóficas, Universidad Nacional Autónoma de México, Ciudad Universitaria, C.P. 04510, \\ Mexico City, Mexico; 'Department of Speech Language Pathology and Audiology, Chung Shan Medical University, Taichung 40201, Taiwan; 'School of \\ Communication Sciences and Disorders, The University of Memphis, Memphis, TN 38152; ${ }^{\mathrm{d}}$ The Institute for Intelligent Systems, The University of Memphis, \\ Memphis, TN 38152; ' Department of Philosophy, The University of Memphis, Memphis, TN 38152; ${ }^{\mathrm{f}}$ Faculty of Law, Humanities and the Art, University of \\ Wollongong, Wollongong, NSW 2522, Australia
}

doi:10.1017/S0140525X16001977. Published by Cambridge University Press, 13 December 2017

Yuna Jhang's affiliation was incorrectly listed in the original online version of this commentary on the target article by Keven \& Akins (2017). The correct affiliations are listed above and have been corrected in the original commentary.

\section{References}

Keven, N. \& Akins, K. A. (2017) Neonatal imitation in context: Sensorimotor development in the perinatal period. Behavioral and Brain Science 40:e381.

Vincini, S., Jhang, Y., Buder, E. H. \& Gallagher, S. (2017) An unsettled debate: Key empirical and theoretical questions are still open. Behavioral and Brain Science 40:e401. https://doi:10.1017/S0140525X16001977. 